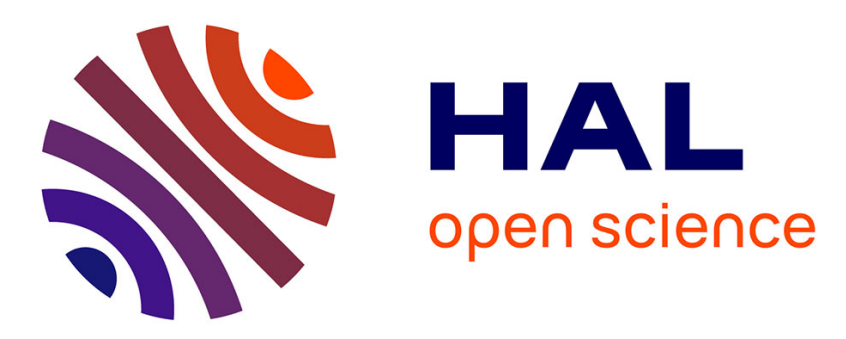

\title{
Study of the asymptotic behaviour of non-linear functionals for the empirical bridge via strong approximations
}

\author{
Corinne Berzin-Joseph, José R. León, Joaquín Ortega
}

\section{- To cite this version:}

Corinne Berzin-Joseph, José R. León, Joaquín Ortega. Study of the asymptotic behaviour of nonlinear functionals for the empirical bridge via strong approximations. Comptes rendus de l'Académie des sciences. Série I, Mathématique, 1998, 327 (7), pp.671-676. 10.1016/S0764-4442(99)80099-8 . hal-00319465

\section{HAL Id: hal-00319465 \\ https://hal.science/hal-00319465}

Submitted on 10 Jan 2019

HAL is a multi-disciplinary open access archive for the deposit and dissemination of scientific research documents, whether they are published or not. The documents may come from teaching and research institutions in France or abroad, or from public or private research centers.
L'archive ouverte pluridisciplinaire HAL, est destinée au dépôt et à la diffusion de documents scientifiques de niveau recherche, publiés ou non, émanant des établissements d'enseignement et de recherche français ou étrangers, des laboratoires publics ou privés. 


\title{
Study of the asymptotic behaviour of non-linear functionals for the empirical bridge via strong approximations
}

\author{
Corinne BERZIN-JOSEPH a , José R. LEÓN ${ }^{b}$, Joaquín ORTEGA ${ }^{\text {c }}$
}

a Département de mathématiques, bâtiment Fermat, Université Versailles-Saint-Quentin-en-Yvelines, 45, avenue des États-Unis, 78035 Versailles cedex, France

E-mail: Corinne.Berzin@math.u-psud.fr

b Escuela de Matemáticas, Facultad de Ciencias, Universidad Central de Venezuela, Paseo Los Ilustres, Los Chaguaramos, Caracas 1041-A, Venezuela

E-mail: jleon@euler.ciens.ucv.ve

c Departamento de matemáticas, IVIC, Apartado Postal 21.827, Caracas 1020-A, Venezuela E-mail: jortega@cauchy.ivic.ve

(Reçu le 5 novembre 1997, accepté le 11 septembre 1998)

\begin{abstract}
We study the asymptotic behaviour of several non-linear functionals of the empirical bridge and obtain some applications to $G$-deviation in density estimation and to Kullback deviation convergence. (C) Académie des Sciences/Elsevier, Paris

Étude du comportement asymptotique de fonctionnelles non-linéaires du pont empirique via des approximations fortes

Résumé. Nous étudions le comportement asymptotique de plusieurs fonctionnelles non linéaires du pont empirique et appliquons ces résultats à la G-déviation de l'estimateur à noyau de la densité et à la convergence pour la déviation de Kullback. (C) Académie des Sciences/Elsevier, Paris
\end{abstract}

\section{Version française abrégée}

Dans cette Note nous étudions le comportement asymptotique de fonctionnelles non linéaires de régularisations du pont empirique. En utilisant un résultat, montré dans une Note précédente [2], pour le même type de fonctionnelles du pont brownien, et un théorème d'approximation forte, nous avons prouvé quelques résultats concernant la déviation d'ordre $p$ et plus généralement une déviation $G$ quelconque définie plus loin et servant dans l'estimation des dérivées d'une densité. Nous précisons le comportement asymptotique du nombre de passages par un niveau pour le processus empirique lissé perturbé défini par Yukich [6], et nous étudions aussi la convergence pour la déviation modifiée de Kullback.

Note présentée par Jean-Pierre KaHANE. 


\section{Berzin-Joseph et al.}

La motivation principale de cette Note est de donner un point de vue unifié pour les problèmes cités auparavant et apparemment non reliés entre eux.

Énonçons les principaux résultats obtenus.

1. Soient $X_{1}, \ldots, X_{n}$ des v.a. i.i.d. de fonction de répartition $F$ à support dans $[0,1]$ et $\mathrm{d} F(u)=s(u) \mathrm{d} u$. L'estimateur à noyau de la densité est défini par : $\widehat{s}_{n}(u)=\frac{1}{h} \int_{-\infty}^{\infty} \varphi\left(\frac{u-v}{h}\right) \mathrm{d} F_{n}(v)$, où $F_{n}$ est la distribution empirique de la suite. Nous définissons comme Yukich [6] le pont empirique régularisé perturbé : $\widetilde{\beta}_{n}^{h}(u)=\sqrt{n}\left(\widehat{F}_{n}(u)-F(u)\right)$, où $\widehat{F}_{n}(u)=\int_{-\infty}^{u} \widehat{S}_{n}(v) \mathrm{d} v$. Yukich a montré que si $\sqrt{n} h^{2} \rightarrow 0$ alors $\widetilde{\beta}_{n}^{h}(u)$ converge en loi vers un $F$-pont brownien $b^{F}(u)$. De plus, en utilisant un développement de Taylor pour $\sqrt{n}\left(\widetilde{F}_{n}(u)-F(u)\right)$, où $\widetilde{F}_{n}(u)=\int_{-\infty}^{\infty} \varphi(v) F(u-h v) \mathrm{d} v$, il obtient que, si $\sqrt{n} h^{2} \rightarrow a$, alors $\widetilde{\beta}_{n}^{h}(u) \rightarrow b^{F}(u)+\frac{a}{2} K_{2} \dot{s}(u)$; ici $K_{2}=\int_{-\infty}^{\infty} \varphi(v) v^{2} \mathrm{~d} v$. En utilisant des résultats antérieurs [2] pour $\widetilde{\mathrm{N}}_{n}^{h}(x)$, le nombre de fois où le processus $\widetilde{\beta}_{n}^{h}$ traverse le niveau $x$ sur l'intervalle de temps $[0,1]$ et pour $\widetilde{\ell}_{n}^{h}(\cdot)$, une modification de la mesure d'occupation pour $\widetilde{\beta}_{n}^{h}$ (voir corollaire 4 pour la définition), nous obtenons le résultat de convergence suivant :

a) si $\sqrt{n} h^{2} \rightarrow 0$, alors $\widetilde{\xi}_{n}^{h}(f)=\frac{1}{\sqrt{h}} \int_{-\infty}^{\infty} f(x)\left(\Lambda_{h} \widetilde{\mathrm{N}}_{n}^{h}(x)-\widetilde{\ell}_{n}^{h}(x)\right) \mathrm{d} x \stackrel{\mathcal{L}}{\longrightarrow} V$, où $\Lambda_{h}=\left(\frac{\pi h}{2\|\varphi\|_{2}^{2}}\right)^{\frac{1}{2}}$, $V$ est une v.a. telle que $\mathcal{L}\left(V \mid \mathcal{F}_{1}\right)=\sigma_{1} \int_{0}^{1} f\left(b^{F}(u)\right) \sqrt{s(u)} \mathrm{d} \widetilde{W}(u)$, où $\mathcal{F}_{1}$ est la $\sigma$-algèbre engendrée par $\left\{b^{F}(u), 0 \leq u \leq 1\right\}, \widetilde{W}(\cdot)$ est un mouvement brownien indépendant de $b^{F}(\cdot)$ (voir théorème 2 dans [2] pour l'énoncé) et $\sigma_{1}$ est défini dans la section 1 ;

b) d'autre part, si $\sqrt{n} h^{2} \rightarrow a$, nous prouvons alors que $\Lambda_{h} \int_{-\infty}^{\infty} f(x) \widetilde{N}_{n}^{h}(x) \mathrm{d} x$ converge en loi vers $\int_{0}^{1} f\left(b^{F}(u)+\frac{a}{2} K_{2} \dot{s}(u)\right) \sqrt{s(u)} \mathrm{d} u$. De plus, nous montrons que $\widetilde{\xi}_{n}^{h}(f)$ converge vers une v.a. $\widetilde{V}$ telle que $\mathcal{L}\left(\widetilde{V} / \mathcal{F}_{1}\right)=\sigma_{1} \int_{0}^{1} f\left(b^{F}(u)+\frac{a}{2} K_{2} \dot{s}(u)\right) \sqrt{s(u)} \mathrm{d} \widetilde{W}(u)$.

2. Nous définissons $\sqrt{n}\left[\widehat{s}_{n}(u)-s_{n}(u)\right]=\frac{1}{h} \int_{-\infty}^{\infty} \varphi\left(\frac{u-v}{h}\right) \mathrm{d} \beta_{n}^{F}(u)$, où $\beta_{n}^{F}(v)=\sqrt{n}\left(F_{n}(v)-F(v)\right)$ est le pont empirique et $s_{n}(u)=E\left(\widehat{s}_{n}(u)\right)$. En intégrant par parties, $\sqrt{n h}\left[\widehat{s}_{n}(u)-s_{n}(u)\right]=\sqrt{h} \dot{\beta}_{n}^{h}(u)$, où $\dot{\beta}_{n}^{h}$ est la dérivée de $\beta_{n}^{h}, \varphi$-régularisation par convolution du pont empirique. Si $s$ posséde deux dérivées il est bien connu que la fenêtre optimale pour le risque quadratique est obtenue pour $h(n)=\mathrm{O}\left(n^{-1 / 5}\right)$, i.e. si $\sqrt{n h} h^{2} \rightarrow C_{0}$. La fenêtre est sous-optimale si $h=\mathrm{o}\left(n^{-1 / 5}\right)$. Il est également bien connu que $\lim _{n \rightarrow \infty} \sqrt{n h}\left[s_{n}(u)-s(u)\right]=c(u)$ pour une fenêtre optimale, où $c(u)$ est une fonction connue, et $\lim _{n \rightarrow \infty} \sqrt{n h}\left[s_{n}(u)-s(u)\right]=0$ pour une fenêtre sous-optimale.

Considérons la fonction $g_{p}(x)=|x|^{p}-E|N|^{p}, p \geq 1$, où $N$ est une v.a. gaussienne standard. Les résultats que nous venons d'énoncer impliquent, pour une fenêtre sous-optimale, le théorème suivant dû à Csörgő et Horváth [3] : $\frac{1}{\sqrt{h}} \int_{0}^{1}\left[\left|\sqrt{n h}\left(\widehat{s}_{n}(u)-s(u)\right)\right|^{p}-E\left[\left|\sqrt{n h}\left(\widehat{s}_{n}(u)-s(u)\right)\right|^{p}\right]\right] \mathrm{d} u$ est équivalent asymptotiquement à : $\frac{1}{\sqrt{h}} \int_{0}^{1} g_{p}\left(\frac{\sqrt{h} \dot{\beta}_{n}^{h}(u)}{\left[\operatorname{var}\left(\sqrt{h} \dot{\beta}_{n}^{h}(u)\right)\right]^{1 / 2}}\right)\left|\sqrt{\operatorname{var}\left(\sqrt{h} \dot{\beta}_{n}^{h}(u)\right)}\right|^{p} \mathrm{~d} u$. En utilisant maintenant le théorème de Komlós, Major et Tusnády (KMT) (voir [4]) nous pouvons remplacer $\dot{\beta}_{n}^{h}(u)$ par la dérivée de la $\varphi$-régularisation de taille $h$ du $F$-pont brownien et obtenir, ainsi, que la dernière intégrale converge vers une loi normale $\mathrm{N}\left(0, \sigma_{p}^{2}\right)$. Un résultat similaire est obtenu pour le cas optimal. Notre approche nous permet également de retrouver le théorème de Beirlant et Mason [1]. En utilisant nos techniques nous considérons aussi ce genre de problème pour les dérivées de la densité et pour une fonction $G$ générale au lieu de $|x|^{p}$.

3. Comme dernière application nous considérons la déviation de Kullback entre l'estimateur à noyau $\widehat{s}_{n}$ et $s$, soit : $\mathcal{K}\left(\widehat{s}_{n}, s\right)=\int_{0}^{1} \widehat{s}_{n}(u) \ln \left(\frac{\widehat{s}_{n}(u)}{s_{n}(u)}\right) \mathrm{d} u$.

Nous montrons que $n \sqrt{h}\left[\mathcal{K}\left(\widehat{s}_{n}, s\right)-\frac{1}{2} \int_{0}^{1} \frac{1}{s(u)} E\left[\widehat{s}_{n}(u)-s(u)\right]^{2} \mathrm{~d} u-\int_{0}^{1}\left(\widehat{s}_{n}(u)-s(u)\right) \mathrm{d} u\right]$ est équivalent asymptotiquement à $\frac{1}{2 \sqrt{h}} \int_{0}^{1} s^{-1}(u)\left[\left|\sqrt{n h}\left(\widehat{s}_{n}(u)-s(u)\right)\right|^{2}-E\left|\sqrt{n h}\left(\widehat{s}_{n}(u)-s(u)\right)\right|^{2}\right] \mathrm{d} u$ 
qui est un cas particulier du résultat précédent, obtenu pour $p=2$, et converge donc vers une loi normale.

\section{Hypotheses and notation}

(H1) Assume the kernel $\varphi$ satisfies: $\int_{-1}^{1} \varphi(t) \mathrm{d} t=1, \varphi \in \mathrm{C}^{1}, \varphi \geq 0$ and has support a subset of $[-1,1]$. Define $\psi(u)=\varphi * \bar{\varphi}(u)$, where $\bar{\varphi}(u)=\varphi(-u)$ and $\theta(u)=\psi(u)\|\varphi\|_{2}^{-2}, u \in \mathbb{R}$. Let $K_{2}=\int_{-\infty}^{\infty} \varphi(v) v^{2} \mathrm{~d} v$. Later, we shall assume that $\varphi$ is even and satisfies additional conditions.

(H2) For the function $f: f \in \mathrm{C}^{2}$ and $\ddot{f}$ is bounded.

(H3) For the function $s: s \in \mathrm{C}^{2}$ on $[0,1], 0<m \leq s(x) \leq M$ for all $x \in[0,1]$.

Further conditions will be required for some applications.

We shall write $l(x)=\sqrt{\frac{\pi}{2}}|x|-1=\sum_{n=1}^{\infty} a_{2 n} H_{2 n}(x)$, where $\left\{H_{n}, n \in \mathbb{N}\right\}$ are the Hermite's polynomials, orthogonal with respect to the standard Gaussian measure $\phi$ and with leading coefficient equal to 1 and $\sigma_{1}^{2}=\int_{-2}^{2} \sum_{n=1}^{\infty} a_{2 n}^{2}(2 n !) \theta^{2 n}(w) \mathrm{d} w$.

\section{Statistical applications}

All the results we are going to state are consequences of Theorem 2 proved in [2]. Indeed in this theorem we study the asymptotic behaviour of non-linear functionals of $\varphi$-regularization by convolution of the $F$-Brownian bridge $b^{F}$ and show that they converge to $\widetilde{W}$, a Brownian motion
independent of $b^{F}$.

The KMT theorem [4] shows that there exists some $F$-Brownian bridge $b_{n}^{F}$ such that $\sup _{t \in[0,1]}\left|\beta_{n}^{F}(t)-b_{n}^{F}(t)\right|=\mathrm{O}\left(\frac{\ln (n)}{\sqrt{n}}\right)$ a.s., and thus, if $\dot{\beta}_{n}^{h}$ (resp. $\dot{b}_{n}^{h}$ ) stands for the derivative of the $\varphi$-regularization $\beta_{n}^{h}$ (resp. $b_{n}^{h}$ ), let $\beta_{n}^{h}(u)=\frac{1}{h} \int_{-\infty}^{\infty} \varphi\left(\frac{u-v}{h}\right) \beta_{n}^{F}(v) \mathrm{d} v$, then $\sup _{t \in[0,1]}\left|\dot{\beta}_{n}^{h}(t)-\dot{b}_{n}^{h}(t)\right|=$ $\mathrm{O}\left(\frac{\ln (n)}{h \sqrt{n}}\right)$. Now, to study the convergence of some particular functionals of $\dot{\beta}_{n}^{h}$, it's enough to use the strong approximation of KMT and Theorem 2 of [2].

Let us state the Corollaries.

\subsection{Convergence in $L^{2}$ for the empirical process}

Let $g$ be a locally Lipschitz function, i.e.

$$
|g(x)-g(y)| \leq|P(x, y)||x-y|
$$

where $P$ is a polynomial.

COROLlaRY 1. - Let $g$ be an even function verifying (1), under $(\mathrm{H} 1),(\mathrm{H} 3)$ and if $h \rightarrow 0, \frac{\ln (n)}{\sqrt{h n}} \rightarrow 0$, $\lim _{n \rightarrow \infty} \int_{0}^{1} g\left(\sqrt{h} \dot{\beta}_{n}^{h}(u)\right) \mathrm{d} u=\int_{0}^{1} E\left\{g\left(\sqrt{s(u)} N\|\varphi\|_{2}\right)\right\} \mathrm{d} u$ in $\mathrm{L}^{2}$.

If $N_{n}^{h}(x)$ stands for the number of crossings for the process $\beta_{n}^{h}$ at level $x$ on $[0,1]$, we have the following:

COROLLARY 2. - Let $f$ be a function verifying $(\mathrm{H} 2)$ and $(\mathrm{H} 3)$ and $(1)$, under $(\mathrm{H} 1)$ and if $h \rightarrow 0, \frac{\ln (n)}{\sqrt{h n}} \rightarrow 0$, then $V_{h}(f)=\Lambda_{h} \int_{-\infty}^{\infty} f(x) \mathrm{N}_{n}^{h}(x) \mathrm{d} x-\int_{-\infty}^{\infty} f(x) \ell_{n}^{h}(x) \mathrm{d} x \rightarrow 0$ in $\mathrm{L}^{2}$, where we define, abusing the notation, $\int_{-\infty}^{\infty} f(x) \ell_{n}^{h}(x) \mathrm{d} x$ as the "occupation measure", $\int_{-\infty}^{\infty} f(x) \ell_{n}^{h}(x) \mathrm{d} x=$
$\int_{0}^{1} f\left(\beta_{n}^{h}(u)\right) \sqrt{s(u)} \mathrm{d} u$. 


\section{Berzin-Joseph et al.}

Remark. - Using this Corollary one has the following convergence

$$
\Lambda_{h} \int_{-\infty}^{\infty} f(x) \mathrm{N}_{n}^{h}(x) \mathrm{d} x \stackrel{\mathcal{D}}{\longrightarrow} \int_{-\infty}^{\infty} f(x) \widetilde{\ell}^{b}(x) \mathrm{d} x .
$$

COROLlaRY 3. - Assume that $f$ verifies (H2) and (1), under (H1) and (H3), if $h \rightarrow 0, \frac{\ln (n)}{\sqrt{n} h} \rightarrow 0$, then $\xi_{n}(f)=\frac{1}{\sqrt{h}} \int_{-\infty}^{\infty} f(x)\left(\Lambda_{h} N_{n}^{h}(x)-\ell_{n}^{h}(x)\right) \mathrm{d} x \stackrel{\mathcal{D}}{\longrightarrow} V$, where $V$ is as in the Introduction.

\subsection{Perturbed empirical process}

$\varphi$ shall stand for an even function until the end of this Note. We can apply the results obtained previously to $\widetilde{N}_{n}^{h}(x)$, the number of crossings at level $x$ on $[h, 1-h]$ for the process $\widetilde{\beta}_{n}^{h}$.

Let us define the following modification of the occupation measure (with a non-rigorous notation as before), $\int_{-\infty}^{\infty} f(x) \widetilde{\ell}_{n}^{h}(x) \mathrm{d} x=\int_{h}^{1-h} f\left(\widetilde{\beta}_{n}^{h}(u)\right) \sqrt{s(u)} \mathrm{d} u$.

COROLlaRY 4. - Assume that $f$ verifies $(\mathrm{H} 2)$ and (1), under $(\mathrm{H} 1)$ and $(\mathrm{H} 3)$, if $h \rightarrow 0, \frac{\ln (n)}{\sqrt{n} h} \rightarrow 0$ and if $\mathrm{s}$ in $\mathrm{C}^{3}[0,1]$, then:

a) if $\sqrt{n} h^{2} \rightarrow 0$, we obtain that $\widetilde{\xi}_{n}(f) \stackrel{\mathcal{D}}{\longrightarrow} V$, where $V$ is the same r.v. that we obtained for the process $\beta_{n}^{h}$ in Corollary 3;

b) on the other hand, if $\sqrt{n} h^{2} \rightarrow a$, then $\tilde{\xi}_{n}(f) \stackrel{\mathcal{D}}{\longrightarrow} \widetilde{V}$, where $\widetilde{V}$ is as in the Introduction.

\section{3. $p$-deviation}

We are interested in the asymptotic behaviour of the $p$ th-order deviation $D_{n}(p)$ studied for the first time by Csörgó and Horvath [3]. Our approach includes the estimation of the derivatives for the density function (see for this the following section):

$$
D_{n}(p)=\frac{1}{\sqrt{h}} \int_{h}^{1-h}\left\{\left|\sqrt{n} h\left(\widehat{s}_{n}(u)-s(u)\right)\right|^{p}-E\left|\sqrt{n h}\left(\widehat{s}_{n}(u)-s(u)\right)\right|^{p}\right\} \mathrm{d} u .
$$

Before stating the following corollary, let us introduce some notations:

where

$$
\begin{gathered}
\sigma_{\mathrm{so}}^{2}=\int_{0}^{1} \sum_{k=2}^{\infty} k !\left[\frac{1}{k !} \int_{-\infty}^{+\infty} H_{k}(y) \phi(y)|y|^{p} \mathrm{~d} y\right]^{2}\left[\int_{-2}^{2} \theta^{k}(\omega) \mathrm{d} \omega\right]\left[\|\varphi\|_{2} \sqrt{s(u)}\right]^{2 p} \mathrm{~d} u, \\
\sigma_{0}^{2}=\int_{0}^{1} \sum_{k=2}^{\infty} k !\left[\frac{1}{k !} \int_{-\infty}^{+\infty} H_{k}(y) \phi(y)\left|y+f_{0}(u)\right|^{p} \mathrm{~d} y\right]^{2}\left[\int_{-2}^{2} \theta^{k}(\omega) \mathrm{d} \omega\right]\left[\|\varphi\|_{2} \sqrt{s(u)}\right]^{2 p} \mathrm{~d} u, \\
\langle K a, a\rangle=\int_{0}^{1} \int_{0}^{1} F(u \wedge v)(1-F(u \vee v)) a(u) a(v) \mathrm{d} u \mathrm{~d} v,
\end{gathered}
$$

$$
\begin{aligned}
a(u)= & \int_{-\infty}^{+\infty}|y|^{p} \phi\left(y-f_{0}(u)\right) \eta(u)^{p-2} \\
& {\left[\left(y-f_{0}(u)\right)^{2} \dot{f}_{0}(u) \eta(u)-\dot{f}_{0}(u) \eta(u)+(p-1)\left(y-f_{0}(u)\right) \dot{\eta}(u)\right] \mathrm{d} y } \\
& f_{0}(u)=\frac{C_{0}}{2} \frac{\ddot{s}(u)}{\|\varphi\|_{2} \sqrt{s(u)}} K_{2} \quad \text { and } \quad \eta^{2}(u)=\|\varphi\|_{2}^{2} s(u) .
\end{aligned}
$$

COROllary 5. - Under (H1) and (H3) if $h \rightarrow 0, \frac{\ln (n)}{\sqrt{n} h} \rightarrow 0$ and if $s$ is in $\mathrm{C}^{3}[0,1]$, then $D_{n}(p) \stackrel{\mathcal{D}}{\longrightarrow} \mathrm{N}\left(0, \sigma_{p}^{2}\right)$, where

$$
\sigma_{p}^{2}=\left\{\begin{array}{cl}
\sigma_{\mathrm{sa}}^{2} & \text { in the sub-optimal case, } \\
\sigma_{0}^{2}+\langle K a, a\rangle & \text { in the optimal case. }
\end{array}\right.
$$


Remark. - In the sub-optimal case the result holds under the less restrictive condition $s \in \mathrm{C}^{2}[0,1]$.

\subsection{Density's derivatives estimation}

Assume that $s$ is in $\mathrm{C}^{m+2}[0,1]$ and satisfies the conditions of Section 1. Define the estimator for the $m$ th-derivative as in [5] by $\widehat{s}_{n}^{(m)}(u)=\frac{1}{n h^{m+1}} \sum_{k=1}^{n} \varphi^{(m)}\left(\frac{u-X_{k}}{h}\right)$, with $\rho \in \mathrm{C}^{m+1}$. We shall use the notation $s_{n}^{(m)}(u)=E\left[\hat{s}_{n}^{(m)}(u)\right]$ and $\sigma_{n, m}^{2}(u)=\operatorname{var}\left(\widehat{s}_{n}^{(m)}(u)\right)^{h}$. It is well known that the optimal estimation window for the mean square error is $h=\mathrm{O}\left(n^{-\frac{1}{2 m+5}}\right)$, i.e. $\sqrt{n} h^{m+\frac{5}{2}} \rightarrow C_{m}$ and in this case

$$
\frac{s_{n}^{(m)}(u)-s^{(m)}(u)}{\sigma_{n, m}(u)} \rightarrow f_{m}(u)=\frac{C_{m} s^{(m+2)}(u) K_{2}}{2\left\|\varphi^{(m)}\right\|_{2} \sqrt{s(u)}}
$$

Let now $G$ be a locally Lipschitz function (see (1) for the definition), we want to study

$$
I_{n}^{(m)}=\frac{1}{\sqrt{h}} \int_{h}^{1-h}\left\{G\left(\frac{\widehat{s}_{n}^{(m)}(u)-s^{(m)}(u)}{\sigma_{n, m}(u)}\right)-E G\left(\frac{\widehat{s}_{n}^{(m)}(u)-s^{(m)}(u)}{\sigma_{n, m}(u)}\right)\right\} \mathrm{d} u .
$$

Let us introduce some notation before stating the next corollary.

$$
\sigma_{\mathrm{so}, m}^{2}=\sum_{k=2}^{\infty} k !\left[\frac{1}{k !} \int_{-\infty}^{+\infty} H_{k}(y) \phi(y) G(y) \mathrm{d} y\right]^{2}\left[\int_{-2}^{2} \theta_{m}^{k}(\omega) \mathrm{d} \omega\right]
$$

with

$$
\begin{gathered}
\theta_{m}(\omega)=\left[\int_{-\infty}^{\infty} \varphi^{(m)}(y) \varphi^{(m)}(y+\omega) \mathrm{d} y\right]\left\|\varphi^{(m)}\right\|_{2}^{-2}, \\
\sigma_{0, m}^{2}=\int_{0}^{1} \sum_{k=2}^{\infty} k !\left[\frac{1}{k !} \int_{-\infty}^{+\infty} H_{k}(y) \phi(y) G\left(y+f_{m}(u)\right) \mathrm{d} y\right]^{2}\left[\int_{-2}^{2} \theta_{m}^{k}(\omega) \mathrm{d} \omega\right] \mathrm{d} u \\
b_{\mathrm{so}}(u)=\left[\int_{-\infty}^{+\infty}-z G(z) \phi(z) \mathrm{d} z\right] \frac{\dot{s}(u)}{2 \sqrt{s(u)}\|\varphi\|_{2} s(u)}, \\
b_{\circ}(u)=\int_{-\infty}^{+\infty} G(y) \phi\left(y-f_{0}(u)\right) \frac{1}{\eta^{2}(u)}\left[\left(y-f_{0}(u)\right)^{2} \dot{f}_{0}(u) \eta(u)-\dot{f}_{0}(u) \eta(u)-\left(y-f_{0}(u)\right) \dot{\eta}(u)\right] \mathrm{d} y
\end{gathered}
$$

(see (3) and (4) for the definitions of $\eta$ and $f_{m}$ ).

CoRollary 6. - Let $G$ be an even function verifying (1), under (H1), (H3), if $h \rightarrow 0, \frac{\ln (n)}{\sqrt{n} h} \rightarrow 0$ and if $s$ is in $\mathrm{C}^{(m+3)}[0,1]$, then $I_{n}^{(m)} \stackrel{\mathcal{D}}{\longrightarrow} \mathrm{N}\left(0, \sigma_{G, m}^{2}\right)$, where,

$$
\begin{aligned}
& \text { if } m \neq 0, \quad \sigma_{G, m}^{2}= \begin{cases}\sigma_{\mathrm{so}, m}^{2} & \text { in the sub-optimal case, } \\
\sigma_{\mathrm{o}, m}^{2} & \text { in the optimal case. }\end{cases} \\
& \text { and if } m=0, \quad \sigma_{G, m}^{2}=\left\{\begin{array}{cl}
\sigma_{\mathrm{so}, m}^{2}+\left\langle K b_{\mathrm{so}}, b_{\mathrm{so}}\right\rangle & \text { in the sub-optimal case, } \\
\sigma_{0, m}^{2}+\left\langle K b_{0}, b_{\mathrm{o}}\right\rangle & \text { in the optimal case. }
\end{array}\right.
\end{aligned}
$$

(See (2) for the definition of $\langle K b, b\rangle$.)

Remark 1. - In the sub-optimal case and since $G$ is even, $b_{\mathrm{so}}(u)=0$ and then $\left\langle K b_{\mathrm{so}}, b_{\mathrm{so}}\right\rangle=0$, so in the particular case $G(x)=|x|^{p}$, we obtain the results proved by Csörgó and Horváth [3]. 


\section{Berzin-joseph et al.}

Remark 2. - In the sub-optimal case, if $m=0$ and since $G$ is even as in the previous section we can drop the hypothesis that $s \in \mathrm{C}^{m+3}$ on $[0,1]$ and use the less restrictive condition $s \in \mathrm{C}^{m+2}$ on $[0,1]$. The same conclusion holds if $m \neq 0$.

\subsection{Kullback deviation}

Consider the "modified" Kullback deviation between the kernel estimator of the density $\widehat{s}_{n}$ and the true density $s$ verifying conditions of Section 2 . Let

$$
\mathcal{K}\left(\widehat{s}_{n}, s\right)=\int_{h}^{1-h} \widehat{s}_{n}(u) \ln \left(\frac{\widehat{s}_{n}(u)}{s(u)}\right) \mathrm{d} u .
$$

We can show this definition makes sense under the condition $h \rightarrow 0, n h^{1+a} \rightarrow+\infty$ for some $a>0$.

Let us now introduce some notation before proving the next corollary:

$$
\begin{gathered}
\sigma_{k}^{2}=\frac{\|\varphi\|_{2}^{4}}{2}\left[\int_{-2}^{2} \theta^{2}(\omega) \mathrm{d} \omega\right], \\
\widetilde{a}(u)=\frac{1}{2 s(u)} \int_{-\infty}^{+\infty} y^{2} \phi\left(y-f_{0}(u)\right) \\
{\left[\left(y-f_{0}(u)\right)^{2} \dot{f}_{0}(u) \eta(u)-\dot{f}_{0}(u) \eta(u)+\left(y-f_{0}(u)\right)\left[\dot{\eta}(u)-\frac{\dot{s}(u)}{\sqrt{s(u)}}\|\varphi\|_{2}\right]\right] \mathrm{d} y}
\end{gathered}
$$

(see (3) for the definitions of $f_{0}$ and $\eta$ ).

Corollary 7. - Under (H1), (H3) and the assumptions, $h \rightarrow 0, n h^{2+a} \rightarrow+\infty$ for some $a>0$, $n \sqrt{h}\left[\mathcal{K}\left(\widehat{s}_{n}, s\right)-\frac{1}{2} \int_{h}^{1-h} \frac{1}{s(u)} E\left[\widehat{s}_{n}(u)-s(u)\right]^{2} \mathrm{~d} u-\int_{h}^{1-h}\left(\widehat{s}_{n}(u)-s(u)\right) \mathrm{d} u \stackrel{D}{\longrightarrow} \mathrm{N}\left(0, \sigma_{2}^{\prime 2}\right)\right.$, where

$$
\sigma_{2}^{\prime 2}=\left\{\begin{array}{cl}
\sigma_{k}^{2} & \text { in the sub-optimal case, } \\
\sigma_{k}^{2}+\langle K \widetilde{a}, \widetilde{a}\rangle & \text { in the optimal case. }
\end{array}\right.
$$

(See (2) for the definition of $\langle K \widetilde{a}, \widetilde{a}\rangle$.)

Remark. - The term $\int_{h}^{1-h}\left(\widehat{s}_{n}(u)-s(u)\right) \mathrm{d} u$ tends to zero more slowly than the required normalization $n \sqrt{h}$, so we could not drop it.

\section{References}

[1] Beirlant J., Mason D.M., On the asymptotic normally of $\mathrm{L}_{p}$-norms of empirical functionals, Math. Meth. Statis. (to appear).

[2] Berzin-Joseph C., León J.R., Ortega J., Increments and crossings for the Brownian bridge: weak convergence (to appear).

[3] Csörgổ M., Horváth L., Central limit theorems for $\mathrm{L}_{p}$-norms of density estimators, Probab. Th. Rel. Fields (1988) 269-291.

[4] Csörgó M., Révész P., Strong approximations in probability and statistics, Academic Press, New York.

[5] Silverman B.W., Weak and strong uniform consistency of the kernel estimate of a density and its derivatives, Ann. Statis. 6 (1978) $177-184$.

[6] Yukich J.E., A. note on limit for perturbed empirical processes, Stoch. Proc. Appl. 33 (1989) 163-173. 